\title{
Spenden für gemeinnützige Zwecke
}

\author{
Pascale Bourquin
}

\section{Relevanz}

Der Staat verwirklicht die gemeinsamen Anliegen der Bürgerinnen und Bürger und erhebt Steuern, um sich zu finanzieren. Der Steuerzahler bleibt einer unter Tausenden. Weder kann er die Höhe der Steuerschuld selbst bestimmen noch kann er sich aussuchen, wofür genau das eigene Geld eingesetzt wird. In Zeiten knapper öffentlicher Budgets ist meist für viele gemeinnütze Zwecke, die vielen Bürgern wichtig sind, nicht genug Geld da. Wie können der Staat und gemeinnützige Organisationen jenseits von Steuern die Bürger für freiwillige Beiträge zur Verwirklichung gemeinnütziger Projekte gewinnen? Was treibt das Spendenverhalten und wie kann mehr Spendenaufkommen erzielt werden? Verdrängen öffentliche Ausgaben die private Spendentätigkeit?

Christian Keuschnigg

\section{Quelle}

Der nachfolgende Text ist eine Zusammenfassung von: Andreoni, James und A. Abigail Payne (2013), Charitable Giving, Handbook of Public Economics Vol. 5, 1-50.

Private Spenden helfen, gemeinnützige Zwecke zu verwirklichen. In den U.S.A. beträgt das Spendenvolumen fast $1,7 \%$ des BIPs, in Deutschland dagegen nur

P. Bourquin $(\square)$

Universität St. Gallen, St. Gallen, Schweiz

E-Mail: p.bourquin@bluewin.ch

(C) Der/die Autor(en) 2018

C. Keuschnigg (Hrsg.), Inklusives Wachstum und wirtschaftliche Sicherheit, https://doi.org/10.1007/978-3-658-21344-2_3 
0,2\% und in Frankreich gar nur 0,14\%. Das Spendenverhalten folgt dabei sehr unterschiedlichen Motivationen und kann abhängig davon auf verschiedene Art und Weise beeinflusst werden. Ein rein altruistisch motivierter Spender ist vorwiegend am Wohlergehen anderer Menschen interessiert und stellt nicht primär die eigene Person in den Vordergrund. Ihn kümmert nur das gemeinnützige Endresultat seiner Spende. Eine zweite Motivation liegt darin, dass Individuen aus Spenden eine innere Zufriedenheit aus der Freude am Geben ableiten, unabhängig davon, welchen Nutzen die Spende beim Empfänger stiftet. Mit solchen selbstbezogenen Präferenzen ziehen die Individuen Nutzen aus dem Akt des Gebens und Helfens und aus der damit verbundenen Anerkennung und dem sozialen Prestige.

Keiner dieser Ansätze kann alle wichtigen empirischen Beobachtungen und Aspekte des gemeinnützigen Spendens allein erklären. In einem Feldexperiment erachteten es nur $40 \%$ der Untersuchten als lohnenswert, einen kleinen Anteil ihres Geldes für den Erwerb von Information über die Produktivität der einzelnen gemeinnützigen Organisationen einzusetzen. Die Teilnehmer des Experiments zogen es vor, ihre Spendenentscheidung ohne diese Informationen zu treffen, obwohl eine weitere Informationsbeschaffung rational gewesen wäre. Unter ökonomischen Gesichtspunkten könnte man bezüglich der Auswahl der Spendenempfänger erwarten, dass Individuen ihre Mittel nur den produktivsten der wohltätigen Vereinigungen zur Verfügung stellen. In der Realität aber verteilen viele Spender ihr Geld auf eine Vielzahl von gemeinnützigen Organisationen. Wenn der zusätzliche Nutzen, welcher mit einer weiteren Geldeinheit für eine gemeinnützige Organisation realisiert wird, mit dem zugewiesenen Betrag fällt, dann werden Individuen mit Freude am Geben ihr gesamtes gespendetes Vermögen lieber auf mehrere Empfänger aufteilen anstatt es auf eine einzige Organisation zu konzentrieren.

Der tatsächliche Erfolg einer gemeinnützigen Organisation hängt ab vom Gesamtbetrag der erhaltenen Spenden, von der Strategie, mit der eine Organisation die Gelder verwendet, sowie von ihrer Produktivität bei der Ressourcenverwendung. Mit dem Erfolg der Organisation ist auch das Prestige des Spenders einem Risiko ausgesetzt. Die Risikoscheu der Spender schafft daher einen ähnlichen Anreiz zur Verteilung der Spenden auf mehrere Empfänger. Risikoaverse Spender bevorzugen es, ein ganzes Portfolio an gemeinnützigen Investitionen zu tätigen und ihre Spenden zu diversifizieren. Diversifikation ermöglicht es ihnen, das Risiko aus dem zufälligen Erfolg einer einzelnen gemeinnützigen Organisation zu vermeiden.

Die Spender sammeln wenige Informationen über die Empfänger und verteilen ihre Spenden gerne über eine Mehrzahl von Projekten. 
Die Kosten des Spendens und folglich auch die relevanten Steueranreize beeinflussen das Spendenvolumen. Der Großteil der empirischen Studien schätzt, dass die absolute Preiselastizität des Spendens etwas mehr als eins beträgt. Das bedeutet, dass eine steuerliche Abzugsfähigkeit von Spenden, welche den Nettopreis für Spender um $1 \%$ verringert, im Durchschnitt die Spenden, die eine berechtigte Organisation erhält, um mindestens $1 \%$ erhöht. Bei der Bereitstellung von gemeinnützigen Gütern und Dienstleistungen sind in diesem Fall Steuersubventionen effizienter als ein direktes staatliches Angebot. Wenn die Regierung z. B. die Ressourcen für Krebsforschung um $100 €$ erhöhen möchte, könnte sie dies direkt mit Staatsausgaben finanzieren und müsste zusätzlich $100 €$ an Steuereinnahmen generieren. Wenn dagegen der Staat die Spenden an förderungswürdige Forschungsinstitute steuerlich absetzbar macht und eine reiche Person mit einem Grenzsteuersatz von $50 \% 100 €$ spenden würde, dann würde ein Steuerausfall von $50 €$ folgen und der Staat müsste lediglich $50 €$ an neuen Steuereinnahmen beschaffen. Das private Engagement ermöglicht es, dass der Staat das gleiche Ziel mit der Hälfte der Kosten für den Steuerzahler erreichen könnte.

Anreize können in unterschiedlichen Formen gesetzt werden und werden daher möglicherweise von Individuen unterschiedlich wahrgenommen. Eine direkte Subvention in Form einer Aufstockung privater Spenden, bei der eine individuelle Spende durch einen Zuschuss von einer Drittperson, Institution oder dem Staat erhöht wird, ist ökonomisch äquivalent zu einer Steuersubvention, bei der ein Teil der Spenden mittels steuerlicher Abzugsfähigkeit zurückerstattet wird. Ein Individuum sollte also auf diese beiden Subventionsarten identisch reagieren. Empirische Belege zeigen aber, dass Spendenzuschüsse 1,2 bis $2 \mathrm{Mal}$ mehr Beiträge erzeugen können als Steuerbegünstigungen. Es existiert ein so genannter „Framing-Effekt“. Menschen legen ein unterschiedliches Verhalten an den Tag, je nachdem, ob sie das Gefühl haben, zu den öffentlichen Ausgaben für gemeinnützige Zwecke aktiv beizutragen oder dem Staat Steuergeld zu entziehen. Steuerabzüge durch Spendenzuschüsse zu ersetzen hat also das Potenzial, das gesamte Spendenvolumen an gemeinnützige Organisationen zu steigern. Die anteilige Subvention von privaten Spenden schafft darüber hinaus eine kooperative Haltung, indem sie Individuen das Gefühl gibt, dass auch andere zum gemeinnützigen Zweck beitragen.

Steuerliche Abzugsfähigkeit senkt die privaten Kosten von Spenden.

Sinken die Kosten um $1 \%$, dann steigt das Spendenaufkommen um mehr als $1 \%$. Anteilige Zuschüsse zu privaten Spenden erhöhen das Spendenaufkommen mehr als gleich hohe Steuerabzüge. 
Die Akteure auf dem Markt für Spenden sind die Spender, welche Geld zur Verfügung stellen, gemeinnützige Organisationen, welche die Mittel nachfragen, die Regierung, die Staatsaufgaben zu erfüllen hat, und Stiftungen, welche als Vermittler agieren. Typischerweise werden Marktteilnehmer auf das Verhalten der anderen Akteure strategisch reagieren. Wenn Individuen rein altruistisch veranlagt wären, müssten staatliche Fördergelder private Spenden eins zu eins verdrängen. Altruistisch motivierte Menschen interessieren sich nur für das Endresultat der Beträge, die sie einer gemeinnützigen Organisation spenden, und dieses Endresultat hängt nur von den gesamten Einnahmen der Organisation ab, und nicht davon, wie sich die Einnahmen auf Spenden und staatliche Fördergelder aufteilen. Im Gegensatz dazu hätten staatliche Fördergelder keinen Einfluss auf private Spenden, wenn Individuen nach selbstbezogenen Präferenzen handeln und nur durch Prestige, soziale Anerkennung und Freude am Geben zum Spenden motiviert würden. Empirische Studien zeigen, dass jeder Dollar staatlicher Fördergelder in einer Reduktion der privaten Spenden von 50 Cents resultiert. Diese Resultate deuten weder auf rein selbstbezogene noch rein altruistische Präferenzen hin und zeigen, dass Individuen ihre Spenden strategisch an die Aktionen der anderen Marktteilnehmer anpassen.

Ein weiterer interessanter Zusammenhang besteht zwischen staatlichen Fördergeldern und gemeinnützigen Fundraising Aktivitäten. Nach empirischen Schätzungen führt eine Erhöhung von öffentlichen Fördergeldern um \$ $1000 \mathrm{zu}$ einem Rückgang der Fundraising Ausgaben um \$137, was wiederum einen Rückgang der Spenden um $\$ 772$ auslöst. Generell führt eine Mehrausgabe für Fundraising von $\$ 1 \mathrm{zu}$ einer marginalen Steigerung von Spenden um ca. \$ 5. Aus dieser Erkenntnis lässt sich ableiten, dass gemeinnützige Organisationen keine Maximierung ihrer Erträge anstreben. Denn wenn sie dies tun würden, müssten sie ihre Fundraising Aktivitäten so lange ausweiten, bis ein zusätzlicher Dollar für Fundraising das Spendenaufkommen um gerade \$ 1 steigert. Eine Erklärung für die tatsächliche Zurückhaltung bei Fundraising Ausgaben könnte sein, dass die Manager von Non-Profit-Organisationen nicht genügende Anreize haben, die Erträge zu maximieren, da sie nicht persönlich an den zusätzlich resultierenden Profiten der Organisation beteiligt sind. Fundraising könnte auch als ,notwendiges Übel“" angesehen werden, da gemeinnützige Organisationen mit einer aggressiven Ausweitung ihrer Fundraising Aktivitäten oft an Ansehen einbüßen. Im Durchschnitt investiert eine gemeinnützige Organisation zwischen $5 \%$ und $25 \%$ der erhaltenen Spenden in weiteres Fundraising. Die Abneigung der Organisationen als auch der Spender gegenüber Fundraising wirft die Frage auf, ob Fundraising sozial optimal oder verschwenderisch und ineffizient ist. 
Ein Euro mehr an öffentlichen Ausgaben für einen gemeinnützigen Zweck reduziert das Spendenaufkommen um 50 Cents. Ein Euro mehr an direkter staatlicher Finanzierung einer gemeinnützigen Organisation mindert ihr Fundraising-Ergebnis um 77 Cents.

Es wäre zu eng gedacht, gemeinnütziges Spenden nur als Ergebnis eines Marktprozesses zu verstehen, wo sich Nachfrage und Angebot treffen. Die Marktdimension blendet wichtige Aspekte aus, welche über die Ökonomie hinausgehen und bis zu den Bereichen der Ethik, Soziologie und Psychologie reichen. Neben strategischen sollte man auch soziale Interaktionen berücksichtigen. Die Kraft des Werbens, Bittens und der Kommunikation sollte bei gemeinnützigen Spenden nicht unterschätzt werden. Empirische Belege zeigen, dass Menschen nicht nur um Fairness bemüht sind, sondern sich eher soziale Anerkennung und eine altruistische Wahrnehmung durch ihre Mitmenschen wünschen. Die Wahrscheinlichkeit einer Spende steigt um 2,2 Prozentpunkte relativ zu einer Basiswahrscheinlichkeit von $26 \%$, wenn der potenzielle Spender von einer ihm nahestehenden oder gleichgesinnten Person angeworben wird. Falls die werbende Person mit dem potenziellen Spender auch gemeinsame Eigenschaften teilt wie z. B. Herkunft, Sportinteressen oder akademische Qualifikation, dann steigt die Wahrscheinlichkeit einer Spende um weitere 2,6 Prozentpunkte. Beim Lobbying für gemeinnützige Organisationen sollte man also Gruppenzugehörigkeit und sozioökonomische Faktoren miteinbeziehen.

Open Access Dieses Kapitel wird unter der Creative Commons Namensnennung 4.0 International Lizenz (http://creativecommons.org/licenses/by/4.0/deed.de) veröffentlicht, welche die Nutzung, Vervielfältigung, Bearbeitung, Verbreitung und Wiedergabe in jeglichem Medium und Format erlaubt, sofern Sie den/die ursprünglichen Autor(en) und die Quelle ordnungsgemäß nennen, einen Link zur Creative Commons Lizenz beifügen und angeben, ob Änderungen vorgenommen wurden.

Die in diesem Kapitel enthaltenen Bilder und sonstiges Drittmaterial unterliegen ebenfalls der genannten Creative Commons Lizenz, sofern sich aus der Abbildungslegende nichts anderes ergibt. Sofern das betreffende Material nicht unter der genannten Creative Commons Lizenz steht und die betreffende Handlung nicht nach gesetzlichen Vorschriften erlaubt ist, ist für die oben aufgeführten Weiterverwendungen des Materials die Einwilligung des jeweiligen Rechteinhabers einzuholen.

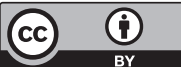

